\title{
Aspirin as an Antifungal-Lock Agent in Inhibition of Candidal Biofilm Formation in Surgical Catheters
}

This article was published in the following Dove Press journal: Infection and Drug Resistance

\author{
Alice Kit Ying Chan \\ Yiu Cheung Tsang \\ Chun Hung Chu (D) \\ Chiu Shun Peter Tsang \\ Faculty of Dentistry, University of Hong \\ Kong, Prince Philip Dental Hospital, Hong \\ Kong Special Administrative Region of \\ the People's Republic of China
}

Background: The antibiotic lock technique (ALT) has been recommended for the prevention and treatment of catheter-related candidaemia. Biofilms of Candida species are resistant to some of the antifungal agents currently used. Aspirin has been shown to have anti-fungal effect but its effect on candidal biofilm is poorly understood.

Purpose: The aim of the current study was to evaluate the anti-biofilm effect of aspirin on Candida biofilms including C. albicans, C. glabrata, C. krusei and C. tropicalis formed on surgical catheters and the concentration and time required to eradicate the biofilms.

Methods: Biofilms of Candida species were grown on silicone catheters and incubated in aspirin at different concentrations for 2, 4 and 24 hours. The biofilms remaining were then determined quantitatively by colony-forming unit (CFU) counts and XTT assays.

Results: The results demonstrated that among the tested Candida species, C. albicans was the most sensitive species towards aspirin. Aspirin at a concentration of $40 \mathrm{mg} / \mathrm{mL}$ in 4 hours was effective in eradicating the biofilm. For all the other tested species, they were eradicated by aspirin at a concentration of $40 \mathrm{mg} / \mathrm{mL}$ in 24 hours.

Conclusion: Our results showed that aspirin may be used as an anti-fungal agent in lock therapy in the treatment of catheter-related candidaemia.

Keywords: Candida species, lock therapy, antifungal, aspirin, catheter-related infection

\section{Introduction}

Catheter-related infections (CRI) are the most common types of nosocomial infections. ${ }^{1}$ The most common pathogens responsible for CRI are coagulase-negative staphylococci, Staphylococcus aureus, and Candida species. ${ }^{2}$ CRI will prolong hospitalization for patients and hence increase the burden in our health care system with each episode costing more than USD $45,000 .^{3}$ It may eventually turn to disseminated diseases leading to end organs disease with heart and eye involvement and hence increase the risk of morbidity and mortality. Candidaemia has been associated with the highest rates of morbidity and mortality in CRIs. ${ }^{4}$ Candidaemia involves the formation of biofilms on implanted devices such as indwelling catheters or on tissue surfaces. ${ }^{5}$

Currently, the management for catheter-related infections caused by Candida species is the removal and replacement of the infected device as well as use of systemic antifungal therapy. ${ }^{2}$ However, in many patients with catheter-related infections, the removal of the infected device is sometimes difficult or has procedure-related risks. ${ }^{2,6}$
Correspondence: Chiu Shun Peter Tsang Faculty of Dentistry, University of Hong Kong, Prince Philip Dental Hospital, 34 Hospital Road, Hong Kong Special Administrative Region of the People's Republic of China

Tel +852-2859-0310

Fax $+852-2858-6144$

Email csptsang@hku.hk
Infection and Drug Resistance 2021:14 |427-1433 
The antibiotic lock technique (ALT) was introduced in the late 1980s and has been recommended for the prevention and treatment of catheter-related infections in specific situations by the Infectious Diseases Society of America (IDSA) and the Centers for Disease Control and Prevention (CDC). ${ }^{7}$ It involves the filling of the catheter lumen with an antimicrobial solution and allowing it to dwell for a time sufficient to sterilize the device. ${ }^{2}$ A review stated that taurolidine, chelator-based antimicrobial and nitroglycerin-citrate-ethanol (NiCE) microbial lock solutions showed promising results in decreasing CRIs with NiCE antimicrobial lock solution gaining specific attention as it does not contain antibiotic and hence not producing bacterial resistance. ${ }^{3}$ Antifungal agents such as caspofungin, micafungin, anidulafungin, lipid formulations of amphotericin B, and fluconazole have been evaluated for their therapeutic effects in ALT for the treatment of Candida spp. associated CRIs. ${ }^{8-10}$ However, biofilms of Candida species normally consist of matrix-enclosed microcolonies of yeasts and hyphae and are resistant to some of the antifungal agents currently in clinical use. ${ }^{11,12}$ Therefore, there is a continuous need for the discovery of new antifungal agents that are effective against Candida biofilms.

Prostaglandins have recently been identified in pathogenic fungi. It was suggested that prostaglandins might be related to the growth of pathogenic filamentous fungi, which could be avoided by prostaglandin $\mathrm{H} 2$ synthase 1 (cyclooxygenase-1) or prostaglandin $\mathrm{G} / \mathrm{G}$ synthase (cyclooxygenase-2) inhibitors. ${ }^{13}$ Acetylsalicylic acid (ASA) is a well-known nonsteroidal anti-inflammatory drug (NSAID) with analgesic, antipyretic and antiinflammatory properties. The pharmacological mechanism of NSAIDs is mediated through the inhibition of cyclooxygenase (COX). ${ }^{14}$ Due to its effect on COX, it has been suggested to be used as an alternative antifungal agent.

Alem and Douglas found that prostaglandin production occurs in candidal planktonic and biofilm growth phenotypes and that prolonged exposure to aspirin may reduce the prostaglandin production in $\sim 25 \%$ for both phenotypes with no growth implications. ${ }^{15}$ ASA significantly decreases C. albicans biofilm formation and reduces viability of biofilm cells at concentrations that could be achieved in humans with therapeutic doses. ${ }^{16}$ Other studies found that ASA also suppresses biofilm formation of C. guilliermondii, C. kefyr, C. glabrata, and C. parapsilosis. ${ }^{17}$

The effectiveness of ALT on Candida species depends on the choice of the antimicrobial agent, the concentration and the treatment duration. Hence, the aim of the current study was to evaluate the effect of aspirin on Candida biofilms formed on surgical catheters. The concentrations and time required for aspirin to eradicate biofilms formed by Candida species including C. albicans, C. glabrata, C. krusei and C. tropicalis were determined.

\section{Materials and Methods Organisms and Culture Conditions}

Four species of Candida species were used in this study. Overnight cultures of C. albicans (ATCC 90028), C. glabrata (ATCC 90030), C. krusei (ATCC 6258) and C. tropicalis (ATCC 13803) were plated on Sabouraud Dextrose Agar (Gibco) plates and incubated at $37^{\circ} \mathrm{C}$.

\section{Aspirin}

Stock solution of aspirin (Sigma) was freshly prepared in dimethyl sulfoxide (DMSO; Sigma) at $1000 \mathrm{mg} / \mathrm{mL}$. Aspirin was diluted in Roswell Park Memorial Institute (RPMI; Gibco) medium supplemented with $2 \%$ glucose at final concentrations ranging from $20 \mathrm{mg} / \mathrm{mL}$ to $45 \mathrm{mg} / \mathrm{mL}$.

\section{Minimal Inhibitory Concentration (MIC) Determination}

The MIC was determined by broth microdilution assay. Candida suspension in RPMI equivalent to a 0.5 McFarland standard was prepared for each isolate. The 96well plate was filled with aspirin $(200 \mu \mathrm{L})$ at different concentrations ranging from $20 \mathrm{mg} / \mathrm{mL}$ to $45 \mathrm{mg} / \mathrm{mL}$ together with $50 \mu \mathrm{L}$ of Candida suspension. It was then incubated for 24 hours at $37^{\circ} \mathrm{C}$ in SpectraMAX 340PC384 (Molecular Devices, US). Absorbance was measured at wavelength of $520 \mathrm{~nm}$. The growth curve was plot at every 30 minutes. The MIC was determined as the lowest concentration that inhibited growth by $50 \%$ compared to that of the positive control. The positive controls were performed with only overnight culture in the wells. Positive controls included DMSO in the same concentration as that in aspirin of $45 \mathrm{mg} / \mathrm{mL}$ were also performed. Each MIC determination was performed in two separate occasions in duplicate.

\section{In vitro Model for Biofilm Formation}

Biofilm was grown on the surface of $100 \%$ silicone catheters (1.5 mm inside and $2.0 \mathrm{~mm}$ outside diameters). Catheters (A-M systems, USA) prepared to a length of $10 \mathrm{~mm}$ were autoclaved. The sections of silicone catheters 
were fully submerged into 96-well plates with $200 \mu \mathrm{L}$ RPMI and $50 \mu \mathrm{L}$ Candida suspension (McFarland standard of $0.5 ; \lambda 520 \mathrm{~nm}$ ). The plate was stored in a shaker incubator of $37{ }^{\circ} \mathrm{C}$ for 48 hours. RPMI supplemented with $2 \%$ glucose was replenished every 24 hours.

\section{Quantitative Measurement of Biofilm}

After 48 hours, the silicone catheters were rinsed gently by phosphate-buffered saline (PBS; $\mathrm{pH}$ 7.2) once to remove the nonadherent cells and then transferred to another fresh 96-well plate containing RPMI and aspirin at the designated test concentrations. During continued incubation in the shaker incubator at $37{ }^{\circ} \mathrm{C}$, the silicone catheters were removed at $2 \mathrm{hr}, 4 \mathrm{hr}$ and $24 \mathrm{hr}$ for quantitative measurement of biofilm removal.

Biofilm growth was quantified colorimetrically by a 2,3-bis(2-methoxy-4-nitro-5isulfophenyl)-2H-tetrazolium -5-carboxanilide (XTT) reduction assay. XTT solution (Sigma, MO, USA) was prepared in phosphate buffered saline (PBS) at a final concentration of $1 \mathrm{mg} / \mathrm{mL}$. Filter sterilized menadione (Sigma, MO, USA) solution $(0.4 \mathrm{mM})$ was also prepared and filtered immediately before each assay. XTT solution of $240 \mu \mathrm{L}$ was added to each well. The plate was then incubated for 3 hours at $37{ }^{\circ} \mathrm{C}$. Positive controls with catheters and cells were prepared. Controls with catheters and cells in DMSO at the same concentration as that in aspirin of $45 \mathrm{mg} / \mathrm{mL}$ were also prepared.

After incubation, the liquid was removed from each well and XTT formazan production was measured by determining the absorbance at $490 \mathrm{~nm}$ in Spectramax. The result was expressed as the percentage of XTT activity remaining relative to the positive controls.

The attached microbial population was also counted using spiral plate technique and was expressed as CFU/ $\mathrm{mL}$. The percentage of remaining viable cells at different concentrations of aspirin relative to the positive controls was calculated. In brief, the catheters were removed from the wells after incubation and rinse with PBS to remove the non-adherent cells. The catheters with the attached biofilms were then vortex mixed in PBS and the cell suspensions were spiral plated on SDA plates for CFU counts. All experiments were performed in two separate occasions in duplicate.

\section{Statistical Analysis}

Statistical analysis was conducted using the one-sample $t$-test to compare the difference among different concentrations within the same species. A $p$-value of $<0.05$ was considered to be statistically significant. The one-way ANOVA was conducted to compare the difference among the four Candida species. A $p$-value of $<0.05$ was considered to be statistically significant.

\section{Results}

The minimal inhibitory concentration (MIC) values of aspirin for C. albicans, C. glabrata, C. krusei and C. tropicalis are $26,28,28$ and $30 \mathrm{mg} / \mathrm{mL}$ respectively. All Candida species were susceptible to aspirin. According to the MIC values, C. albicans was the most sensitive species against aspirin.

Results from the biofilm experiment showed that the anti-biofilm effect of aspirin on Candida species is concentration-dependent as shown in Figure 1A-D and Figure 2A-D. The minimal biofilm eradication concentration (MBEC) $(\mathrm{mg} / \mathrm{mL})$ over a $24-\mathrm{h}$ exposure time that the growth of Candida species was undetected (the percentage remaining of viable count $<0.5$ ) was $40 \mathrm{mg} / \mathrm{mL}$ (Figure $1 \mathrm{~A}-\mathrm{D})$. The percentage remaining in viable count of C. albicans, C. glabrata, C. krusei and C. tropicalis in aspirin at $4 \mathrm{~h}$ was $0.2,1.4,2.3$ and 2.7 , respectively (Figure 1A-D), whereas \% XTT activities remaining at this concentration were $3.8,0.5,31.6$ and 13 for C. albicans, C. glabrata, C. krusei and C. tropicalis, respectively (Figure 2A-D) $(p<0.001)$. Lower exposure time $(2 \mathrm{~h})$ showed a higher percentage remaining in viable counts at concentration of $45 \mathrm{mg} / \mathrm{mL}(0.8,27.9,16.9$ and 1.3 for C. albicans, C. glabrata, C. krusei and C. tropicalis, respectively) (Figure 1A-D).

According to the MIC and MBEC values, the biofilm cultures of the tested Candida species were more resistant to the effect of aspirin when compared with the planktonic culture. In the positive control group, the viable cell counts of Candida species ( $>10^{5} \mathrm{CFU}$ ) were maintained until the end of the study that confirmed the viability of the cells (Figure 1A-D).

\section{Discussion}

Formation of biofilm on medical devices such as central venous catheters can cause CRIs. Staphylococci such as Staphylococcus aureus and Candida species were the most common pathogens of CRI. The management of CRIs involves a multilevel approach involving catheter management such as salvage, exchange or removal together with empirical systemic antimicrobial therapy. The Infectious Disease Society of America recommends to remove catheter for any CRIs due to candidemia together with systemic 

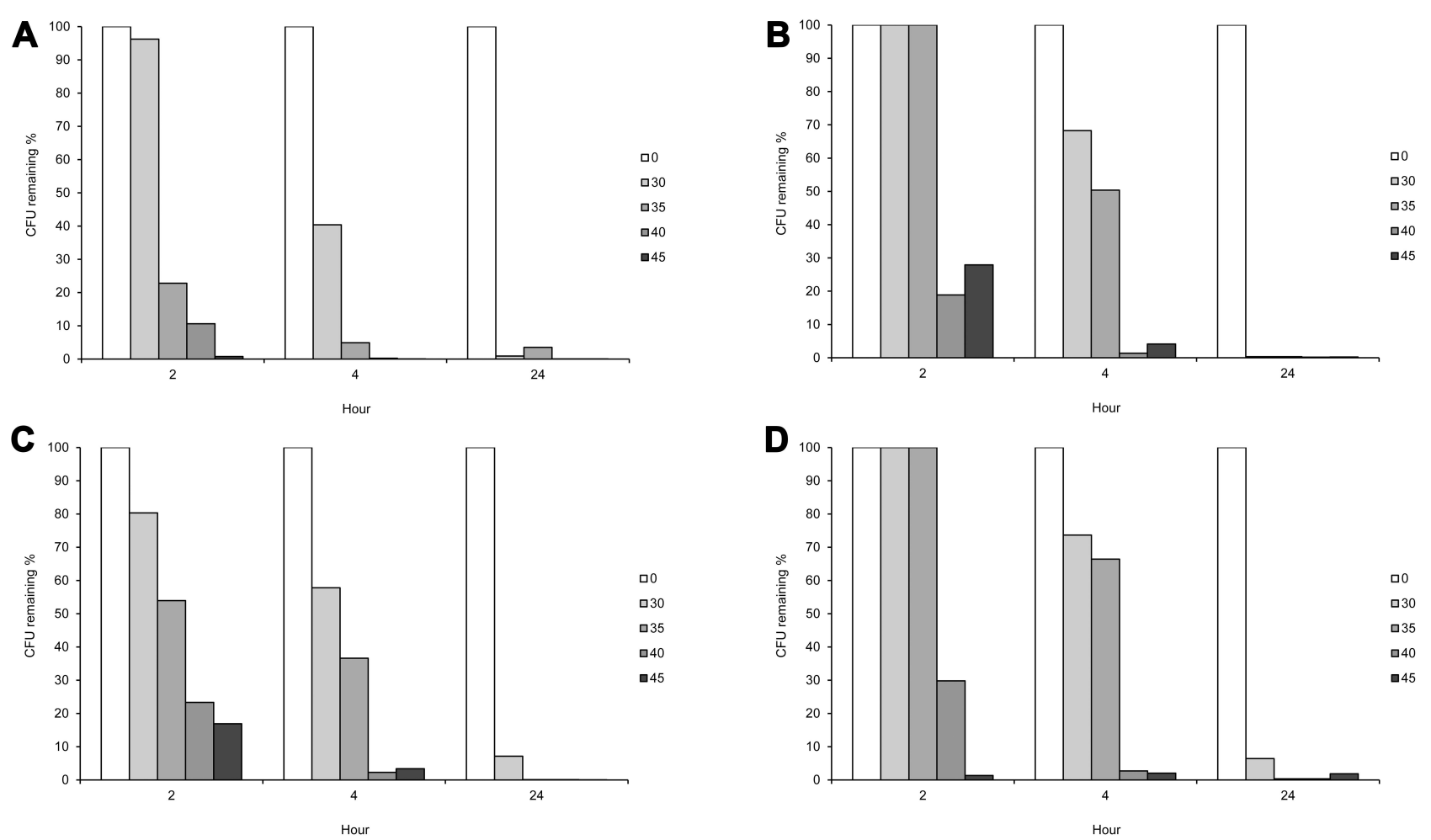

Figure I CFU percentage remaining (\%) of Candida species at different concentrations of aspirin (mg/mL) in 2, 4 and 24 hours. (A) Candida albicans. (B) Candida glabrata. (C) Candida krusei. (D) Candida tropicalis.

antifungal treatment. ${ }^{18}$ With the introduction of ALT, CRIs can be treated in a simpler way without the removal of catheters and use of systemic antifungal therapy. ALT is an option in current guideline for the management of catheter-related bacteraemia, but it is not yet a recognized option for catheter-related candidaemia., ${ }^{2,6}$ However, there have been a number of studies showing the efficacy of some antifungal agents in ALT.

Candida species has shown resistance to the azole group of antifungal agents. Current studies show that caspofungin and amphotericin B are effective in treating catheter-related candidaemia. Ko et al concluded that limited duration of fungal ALT (less than five days) using amphotericin B and caspofungin is sufficient to treat the infected catheters, which will decrease the risk of resistance and also the cost in clinical practice. ${ }^{19}$ It is necessary to discover more antifungal drugs in order to overcome the resistance problem. Aspirin has been shown to have an antifungal effect. ${ }^{20}$ Currently, in vitro assessment of the antibiofilm activities of aspirin on Candida species were limited. . $^{16,17,20,21}$

In the current study, $C$. albicans showed the highest viable cell count and XTT activities while $C$. krusei has the lowest without the addition of aspirin. This demonstrated that C. albicans is a better biofilm former on silicone tubing than the other Candida species. This finding agrees with a previous study that $C$. albicans produced more biofilm on polyvinyl chloride disks than other Candida species as determined by dry-weight, colorimetric, or radioisotope assays. ${ }^{22}$

From our result, aspirin at a concentration of $40 \mathrm{mg} /$ $\mathrm{mL}$ for at least 4 hours $(p<0.001)$ was required to eradicate the $C$. albicans biofilm (Figure 1A) whereas biofilm of all the other Candida species was eradicated after 24 hours by aspirin at a concentration of $40 \mathrm{mg} / \mathrm{mL}$ $(p<0.001)$ (Figure 1B-D). This suggested that aspirin at a concentration of $40 \mathrm{mg} / \mathrm{mL}$ locked for 24 hours could be used as the antifungal agent in Candida related CRI. In Ko's et al study, five days were required to eradicate the biofilm using amphotericin B and caspofungin as opposed to one day in the current study. ${ }^{19}$ The use of aspirin therefore may have the advantages of reducing the risk of drug resistance development and the cost of clinical practice in the future.

In our study, the result obtained is different from some previous studies. ${ }^{16,17,21} \mathrm{Al}$-Bakri et al found that the MIC and the MBEC values for C. albicans were 2.65 and $3.83 \mathrm{mg} / \mathrm{mL}$, respectively. ${ }^{21}$ Stepanovic et al and Alem 

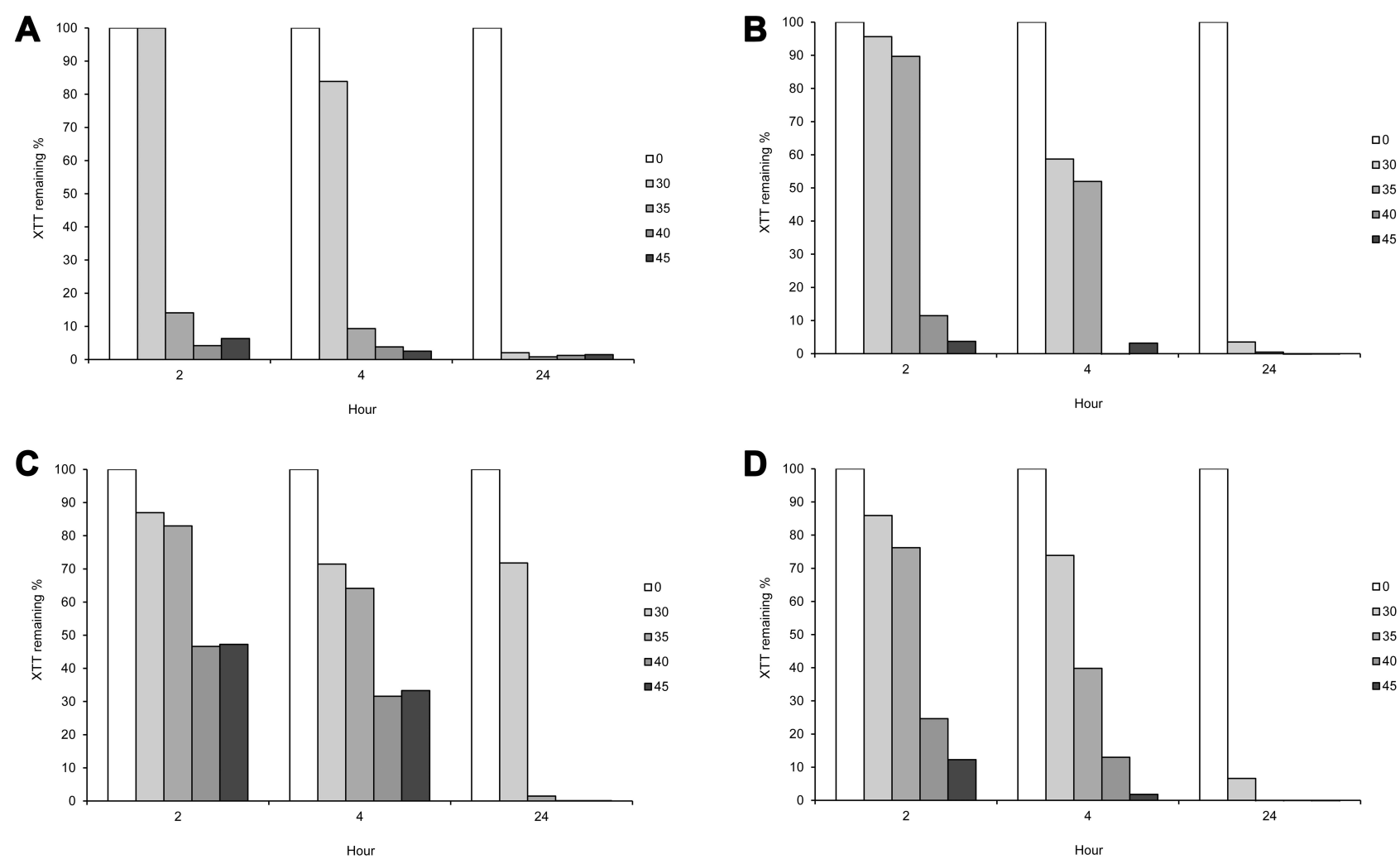

Figure 2 XTT activity percentage remaining (\%) of Candida species at different concentrations of aspirin (mg/mL) in 2, 4 and 24 hours. (A) Candida albicans. (B) Candida glabrata. (C) Candida krusei. (D) Candida tropicalis.

and Douglas found that the MIC value for C. albicans were lower than $1 \mathrm{mg} / \mathrm{mL}^{16,17}$ The difference can be due to the following reasons.

Different methods used in determination of antibiofilm effect gives different results. Some studies used either viable cell count or XTT method or both. The XTT method measured the metabolic activities of the cells and may be positively related with viable cell counts. ${ }^{23}$ In most studies, $50 \%$ reduction of XTT values was used as a guide for effective anti-biofilm activities while the complete eradication of biofilm was used in the viable cell counting method. In our study, the MBEC results obtained from viable cell count and XTT values with over $99 \%$ reduction were comparable.

Yeast nitrogen base (YNB) with glucose and RPMI with glucose are commonly used as the culture medium for Candida species. In our study, aspirin could not be dissolved in YNB with the formation of precipitates. RPMI with glucose was used instead. However, aspirin could not be well dissolved in RPMI once the concentration was higher than $50 \mathrm{mg} / \mathrm{mL}$. Therefore, aspirin concentrations ranging from $20 \mathrm{mg} / \mathrm{mL}$ to $45 \mathrm{mg} / \mathrm{mL}$ were used. It has been shown that the MIC values for $C$. albicans in YNB were slightly different from those obtained in RPMI medium, ${ }^{24}$ therefore partly explains the different results observed in different studies.

All Candida species used in our study were reference strains and only one strain from each Candida species was used in our study. The efficacy of ALT may be straindependent and species-dependent as shown in our results and in another study. ${ }^{25}$ Therefore, more strains and species such as $C$. parapsilosis should be included in further studies.

In conclusion, our study demonstrated that among the four Candida species, C. albicans, C. glabrata, C. krusei and $C$. tropicalis, $C$. albicans was the most sensitive species towards aspirin in which $C$. albicans biofilm was eradicated by aspirin at a concentration of $40 \mathrm{mg} / \mathrm{mL}$ in 4 hours. For all the tested species, they were eradicated by aspirin at a concentration of $40 \mathrm{mg} / \mathrm{mL}$ in 24 hours. Our results showed that aspirin may be used as an anti-fungal agent in lock therapy in the treatment of catheter-related candidaemia. However, it is desirable to have a short dwell time for its use in the intravenous catheter. Besides, further study can be conducted to investigate if aspirin can provide a prolong protection to prevent the recurrence of 
biofilm. Subsequent in vivo studies can be conducted to develop guidelines for clinical use of aspirin as an antifungal agent in the future.

\section{Acknowledgments}

This study was supported by RGC General Research Fund (17100820). The authors would like to thank Ms. Joyce Yau, Mr. Alan Wong and Mr. Y.Y. Chui for laboratory and technical assistance and Mr. Shadow Yeung for the statistical analyses.

\section{Disclosure}

The abstract of this paper was presented at the 25th IADR Southeast Asian Division Meeting as a poster presentation with interim findings. The poster's abstract was published in "Poster Abstracts"in IADR Abstract Archives: https:// iadr.abstractarchives.com/abstract/sea11-153200/aspirin-as -an-antifungal-lock-therapy-agent. The authors report no conflicts of interest in this work.

\section{References}

1. Canton-Bulnes ML, Garnacho-Montero J. Practical approach to the management of catheter-related bloodstream infection. Rev Esp Quimioter. 2019;32(Suppl 2):38-41.

2. Mermel LA, Allon M, Bouza E, et al. Clinical practice guidelines for the diagnosis and management of intravascular catheter-related infection: 2009 update by the Infectious Diseases Society of America. Clin Infect Dis. 2009;49(1):1-45.

3. Malek AE, Raad II. Preventing catheter-related infections in cancer patients: a review of current strategies. Expert Rev Anti Infect Ther. 2020;18(6):531-538. doi:10.1080/14787210.2020.1750367

4. Wenzel RP. Nosocomial candidemia: risk factors and attributable mortality. Clin Infect Dis. 1995;20(6):1531-1534. doi:10.1093/clinids/20.6.1531

5. Douglas LJ. Candida biofilms and their role in infection. Trends Microbiol. 2003;11(1):30-36. doi:10.1016/S0966-842X(02)00002-1

6. Chaves F, Garnacho-Montero J, Del Pozo JL, et al. Diagnosis and treatment of catheter-related bloodstream infection: clinical guidelines of the Spanish Society of Infectious Diseases and Clinical Microbiology and (SEIMC) and the Spanish Society of Spanish Society of Intensive and Critical Care Medicine and Coronary Units (SEMICYUC). Med Intensiva. 2018;42(1):5-36. doi:10.1016/j. medin.2017.09.012

7. O'Grady NP, Alexander M, Dellinger EP, et al. Guidelines for the prevention of intravascular catheter-related infections. Centers for Disease Control and Prevention. MMWR Recomm Rep. 2002;51(RR10):1-29.

8. Cateau E, Rodier MH, Imbert C. In vitro efficacies of caspofungin or micafungin catheter lock solutions on Candida albicans biofilm growth. J Antimicrob Chemother. 2008;62(1):153-155. doi:10.1093/ jac/dkn160
9. Walraven CJ, Lee SA. Antifungal lock therapy. Antimicrob Agents Chemother. 2013;57(1):1-8. doi:10.1128/AAC.01351-12

10. Imbert C, Rammaert B. What could be the role of antifungal lock-solutions? From bench to bedside. Pathogens. 2018;7(1):6. doi:10.3390/pathogens7010006

11. Hawser SP, Douglas LJ. Resistance of Candida albicans biofilms to antifungal agents in vitro. Antimicrob Agents Chemother. 1995;39 (9):2128-2131. doi:10.1128/AAC.39.9.2128

12. Chandra J, Mukherjee PK, Leidich SD, et al. Antifungal resistance of candidal biofilms formed on denture acrylic in vitro. $J$ Dent Res. 2001;80(3):903-908. doi:10.1177/00220345010800031101

13. Noverr MC, Toews GB, Huffnagle GB. Production of prostaglandins and leukotrienes by pathogenic fungi. Infect Immun. 2002;70 (1):400-402. doi:10.1128/IAI.70.1.400-402.2002

14. Vane JR. Inhibition of prostaglandin synthesis as a mechanism of action for aspirin-like drugs. Nat $N$ Biol. 1971;231(25):232-235. doi:10.1038/newbio231232a0

15. Alem MA, Douglas LJ. Prostaglandin production during growth of Candida albicans biofilms. J Med Microbiol. 2005;54 (11):1001-1005. doi:10.1099/jmm.0.46172-0

16. Alem MA, Douglas LJ. Effects of aspirin and other nonsteroidal anti-inflammatory drugs on biofilms and planktonic cells of Candida albicans. Antimicrob Agents Chemother. 2004;48(1):41-47. doi:10.1128/AAC.48.1.41-47.2004

17. Stepanovic S, Vukovic D, Jesic M, Ranin L. Influence of acetylsalicylic acid (aspirin) on biofilm production by Candida species. $J$ Chemother. 2004;16(2):134-138. doi:10.1179/joc.2004.16.2.134

18. Malek A, Raad I. Catheter- and device-related infections in critically ill cancer patients. In: Nates JL, Price KJ, editors. Oncologic Critical Care. Springer International Publishing; 2020:1401-1417.

19. Ko KS, Lee JY, Song JH, Peck KR. In vitro evaluation of antibiotic lock technique for the treatment of Candida albicans, C. glabrata, and C. tropicalis biofilms. J Korean Med Sci. 2010;25(12):1722-1726. doi: $10.3346 / \mathrm{jkms} .2010 .25 .12 .1722$

20. Abdelmegeed E, Shaaban MI. Cyclooxygenase inhibitors reduce biofilm formation and yeast-hypha conversion of fluconazole resistant Candida albicans. J Microbiol. 2013;51(5):598-604. doi:10.1007/s12275-013-3052-6

21. Al-Bakri AG, Othman G, Bustanji Y. The assessment of the antibacterial and antifungal activities of aspirin, EDTA and aspirin-EDTA combination and their effectiveness as antibiofilm agents. J Appl Microbiol. 2009;107(1):280-286. doi:10.1111/j.13652672.2009.04205.x

22. Hawser SP, Douglas LJ. Biofilm formation by Candida species on the surface of catheter materials in vitro. Infect Immun. 1994;62 (3):915-921. doi:10.1128/IAI.62.3.915-921.1994

23. Ferreira JA, Carr JH, Starling CE, de Resende MA, Donlan RM. Biofilm formation and effect of caspofungin on biofilm structure of Candida species bloodstream isolates. Antimicrob Agents Chemother. 2009;53(10):4377-4384. doi:10.1128/AAC.00316-09

24. Soustre J, Rodier MH, Imbert-Bouyer S, Daniault G, Imbert C. Caspofungin modulates in vitro adherence of Candida albicans to plastic coated with extracellular matrix proteins. J Antimicrob Chemother. 2004;53(3):522-525. doi:10.1093/jac/dkh099

25. Deva R, Ciccoli R, Kock L, Nigam S. Involvement of aspirin-sensitive oxylipins in vulvovaginal candidiasis. FEMS Microbiol Lett. 2001;198(1):37-43. doi:10.1111/j.1574-6968.2001. tb10616.x 


\section{Publish your work in this journal}

Infection and Drug Resistance is an international, peer-reviewed openaccess journal that focuses on the optimal treatment of infection (bacterial, fungal and viral) and the development and institution of preventive strategies to minimize the development and spread of resistance. The journal is specifically concerned with the epidemiology of

Submit your manuscript here: https://www.dovepress.com/infection-and-drug-resistance-journa| antibiotic resistance and the mechanisms of resistance development and diffusion in both hospitals and the community. The manuscript management system is completely online and includes a very quick and fair peerreview system, which is all easy to use. Visit http://www.dovepress.com/ testimonials.php to read real quotes from published authors. 